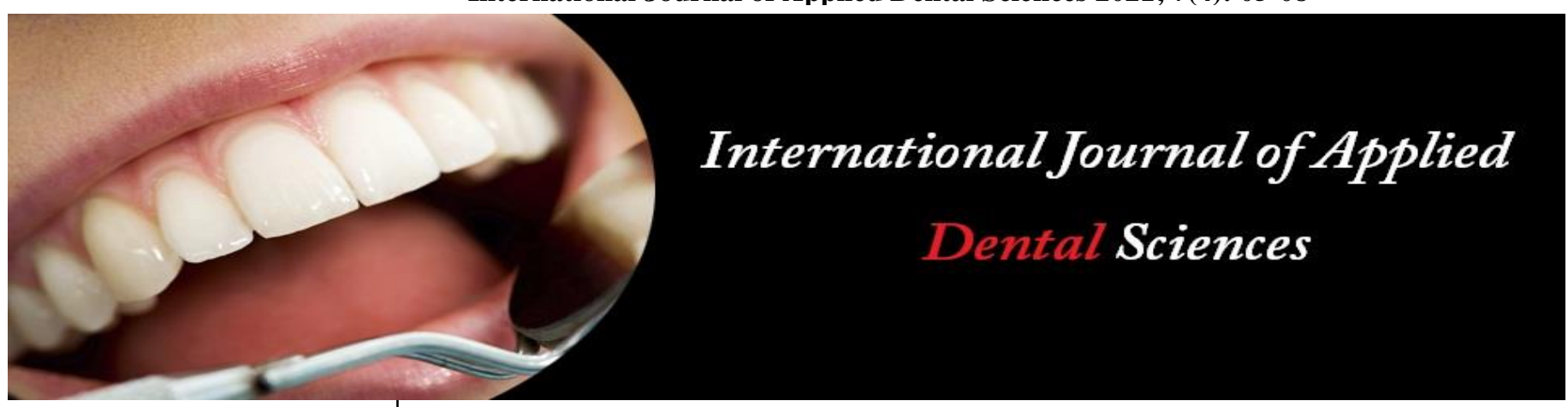

ISSN Print: 2394-7489

ISSN Online: 2394-7497

IJADS 2021; 7(4): 05-08

(C) 2021 IJADS

www.oraljournal.com

Received: 02-08-2021

Accepted: 04-09-2021

Dr. Saket Mehrotra

MBBS, MD, Reader and HOD,

Pathology, Sardar Patel

Postgraduate Institute of Dental

and Medical Sciences, Lucknow,

Uttar Pradesh, India
Corresponding Author:

Dr. Saket Mehrotra

MBBS, MD, Reader and HOD,

Pathology, Sardar Patel

Postgraduate Institute of Dental and Medical Sciences, Lucknow,

Uttar Pradesh, India

\section{Onset of urinary tract infections in ICU patients}

\section{Dr. Saket Mehrotra}

DOI: $\underline{\text { https://doi.org/10.22271/oral.2021.v7.i4a.1343 }}$

\section{Abstract}

Background: Urinary tract infections are widespread among intensive care unit patients than in the general population. But what makes the occurrence of UTIs more common in ICU patients? Although studies about the prevalence of urinary tract infections are available, significantly less data is available on the onset of UTIs in intensive care units. Therefore, we aimed this study to evaluate the prevalence of urinary tract infections in ICU patients. We also tried to find out different risk factors and their possible solutions.

\section{Aims and Objectives}

- Risk factors involved in the onset of urinary tract infections in ICU patients

- To make recommendations based on the study

Materials and Methods

The primary target audience in our research work is particularly, i.e. intensive care unit patients. Therefore, we decided to collect data from 3 intensive care units from three hospitals. We also included a questionnaire for physicians to know their points of view about the issue. Finally, we collected data and compiled it in MS Excel for further processing.

Results: The prevalence of the population was found to be very high. Almost forty per cent was recorded.

Conclusion: Cleanliness, hygiene and proper sterilization is needed and is the need of the hour.

Keywords: UTI, catheter, ICU, prevalence.

\section{Introduction}

Urinary tract infection (UTI) is defined as the infection of any part of the urinary system, including kidneys, urethra, ureters, and bladder. UTIs are considered among the severe public health issues and many gram-negative and gram-positive bacteria are involved (Flores, 2015) ${ }^{[1]}$. It affects about 150 million people in the world each year (May et al., 2016) ${ }^{[2]}$. Escherichia coli, Klebsiella pneumonia, Proteus mirabilis, Enterococcus faecalis and Staphylococcus saprophyticus are commonly found in uro-pathogens. Among all these pathogens, E. coli is most predominant in urinary tract infections (Alanazi, 2018) ${ }^{[3]}$. E. coli causes $41.4 \%$ of urinary tract infections worldwide (Anwar et al., 2018), while the prevalence of E. coli as uropathogen in Pakistan is about 33.3\% (Jihar et al., 2018). The occurrence of urinary tract infections (UTIs) in the patients admitted in intensive care units (ICUs) is more common than in the general population (Anwaar et al., 2018). It is thought to be because of many sources of infection and their exposure to ICU patients (Liu et al., 2018) ${ }^{[6]}$.

Common antibiotics used as a drug of choice for the treatment of UTIs include co-trimoxazole, nitrofurantoin, ciprofloxacin ampicillin and carbapenems (Alanazi., 2018) ${ }^{[3]}$. However, different studies on these drugs showed that many drugs are not helpful anymore against uropathogens because they have developed against these drugs (Sotto et al., 2001) ${ }^{\text {[7]. This }}$ situation of increasing prevalence and antibiotics resistance is becoming a threat to the country's economy and society (Flores, 2015) ${ }^{[1]}$.

\section{Rationale of Study}

Lee et al. (2016) ${ }^{[10]}$ and Liu et al. (2018) ${ }^{[6]}$ conducted a study to elaborate on different risk factors for urinary tract infections in intensive care unit patients. For this study, they compared UTI cases of ICU patients with an uninfected control group. 
It was observed that ICU patients tended to get urinary tract infections soon after their admission to the intensive care unit. Even prevalence was even more common in surgical patients. Among observed risk factors, catheter placement in the emergency and the operating room was found most crucial risk factor.

Jiménez et al. (2015) conducted a similar study to identify and evaluate different risk factors and antibiotic resistance in urinary tract infections. They reported that catheter-associated urinary tract infections (CAUTIs) are the most common nosocomial acquired infections, with high antibiotic resistance levels. They said on the basis of their experiment carried out at a urology department of a hospital. The report shows that CAUTIs are severe complications found in hospitalized patients and may cause higher costs. It was observed that the incidence of CAUTI was $8.2 \%$ in patients who had a catheter.

Urinary tract infections (UTIs) are now very fast-spreading infections around the globe, especially in under-developed countries. One of the most critical risk factors for UTIs is the use of non-sterilized equipment and catheters in intensive care units. At the same time, drug resistance is also very much common among ICU patients due to the use of multiple drugs with heavy doses.

Although studies are available about the prevalence of urinary tract infections but significantly less data is available on the onset of UTIs in intensive care units. We aimed this study to evaluate the prevalence of urinary tract infections in ICU patients. We also tried to find out different risk factors and their possible solutions.

\section{Objectives}

Every research is carried out to meet some already set objectives. These objectives are based on the area of the study and different focus points. Objectives of any research study decide the goals and milestones of that research work. We focused on the following purposes in the current study.

- To find the prevalence of urinary tract infections in the patients admitted to intensive care units

- Risk factors involved in the onset of urinary tract infections in ICU patients

- Steps carried out by hospitals to reduce the risk

- To make recommendations on the basis of study

We tried our best to achieve all of these objectives and we succeeded very much. The results will be discussed in the following chapters.

\section{Methodology}

The required relevant data was collected through different techniques of data collection that include surveys and questionnaires. All the collected data will be compiled for proper analysis. We will provide the final results of this research to the public domain so that everyone may have a straightforward approach to the results. This research will facilitate the management to understand the risk factors and improvement areas for patients admitted in intensive care units to minimize the onset of urinary tract infections.

\section{Sample}

For ease and convenience, a sample is selected to study the population that is considered as the representative of a population. A sample is part of the population and is checked for the outcomes and findings of the whole population (Speak et al., 2018). A sampling of the entire population is a pretty convenient way to study a population, especially when it is quite impossible to check the whole population. However, for better and unbiased results, samples must be selected with proper sampling techniques. Among many sampling techniques, we convenience sampling chosen for our research study because of flexibility and no exclusion and inclusion criteria (Speak et al., 2018).

The primary target audience in our research work is particularly, i.e. intensive care unit patients. We decided to collect data from 3 intensive care units from three hospitals. We also included a questionnaire for physicians to know their points of view about the issue. We collected data and compiled it in MS Excel for further processing.

\section{Results}

We selected three hospitals to collect data of their intensive care units about the prevalence of urinary tract infections (UTIs). A total of 798 intensive care unit patients were considered from all selected hospitals. We collected data and segregated it into two categories, i.e. urinary tract infection positive patients and urinary tract infection negative patients. Among these total 798 patients, we found that 319 (39.97\%) patients developed urinary tract infections after their admission to ICU. At the same time, other 479 (60.01\%) were found negative during hospital tests for urinary tract infections. After complete data collection of all the patients, we compiled and arranged data by using MS Excel for further processing.

The following graphs and tables show the results of all aimed parameters related to our selected topic.

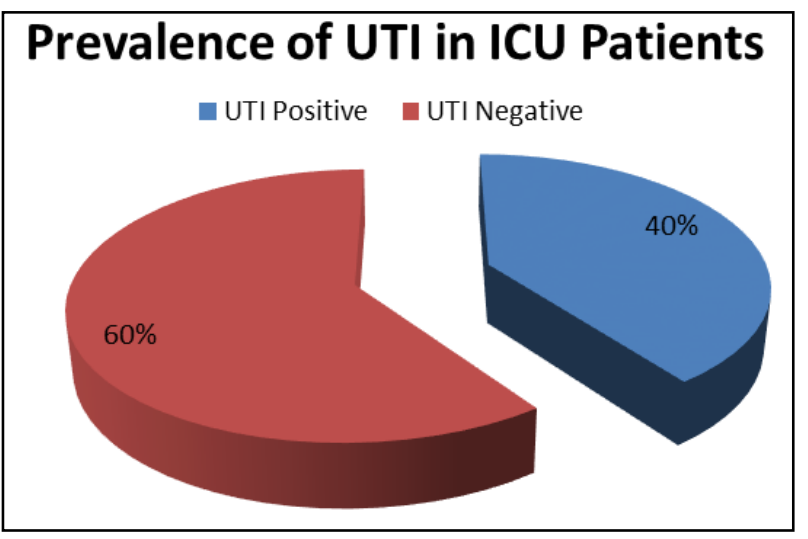

Fig 1: Prevalence of Urinary Tract Infections in ICU Patients

We also segregated data on a gender and age basis to refine the results of our study. Below figures and tables below show further results of the study.

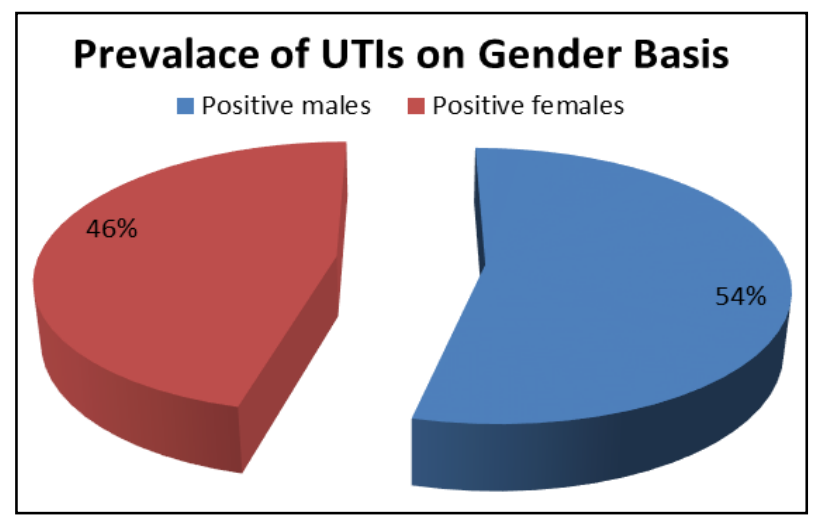

Fig 2: Prevalence of Urinary Tract Infections (UTIs) on Gender Basis

It was recorded in the results of the present study that urinary 
tract infection was found in all age groups. The youngest UTI positive patient in recorded data was just 23 years old, while the most geriatric UTI positive patient in ICU was 81 years of age. The prevalence percentage of urinary tract infections in different age groups are shown in Fig. 3.

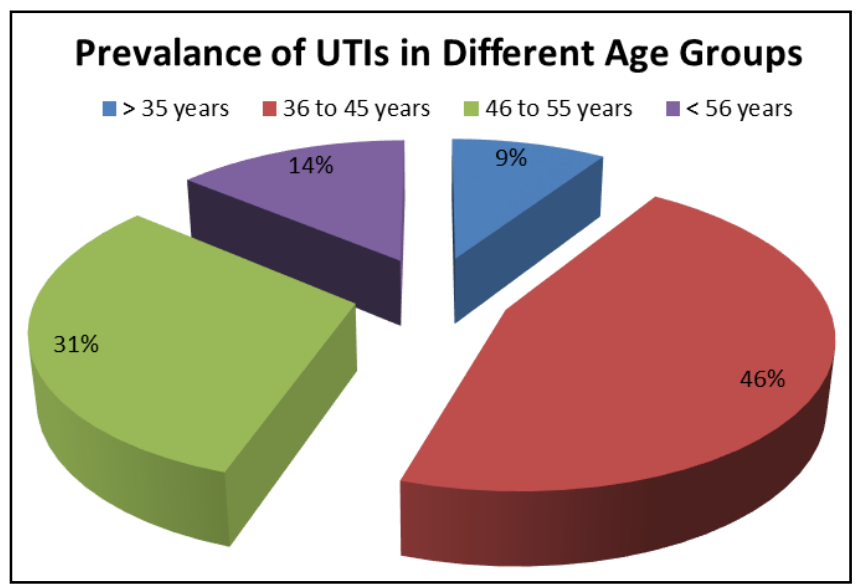

Fig 3: Prevalence of Urinary Tract Infections (UTIs) in Different Age Group

\section{Risk Factors for Urinary Tract Infections in ICU}

We met with physicians of the same hospitals and discussed the risk factors involved in the high incidence of urinary tract infection in ICU patients. As a result, we concluded the following risk factors that play a role in developing UTIs in patients admitted to intensive care units.

- Catheters - the most prevalent issue in this regard: The incidence of urinary tract infections is so much high due to catheters that UTIs are often called catheter-associated urinary tract infections (CAUTIs). It is maybe due to non-sterile catheters or wrong insertion by unskilled medical assistants.

- Surgical equipment - they need to be properly sterilized before their use and must be appropriately disposed of after their usage.

- Non-hygienic environment - another significant risk factor for urinary tract infections in intensive care units. The non-hygienic environment helps the germs to move from one patient to another patient in intensive care units.

- Diagnostic failures - it is significantly less in ICUs, but it still occurs when some physicians overlook or ignore some symptoms of the admitted patients that may lead to serious health issues, including urinary tract infections.

\section{Discussion}

The prevalence of urinary tract infections is much higher among intensive care unit patients when compared with the incidence of UTIs in general populations (Duszyńska et al., 2016) ${ }^{[8]}$. In our research, among 798 patients of intensive care unit, $319(39.97 \%)$ patients developed urinary tract infection while other $479(60.01 \%)$ were negative for urinary tract infections (UTIs). As the already available research studies showed, the use of catheters and surgical instruments (which may not be sterilised appropriately) present the most significant risk factor for the development of UTIs among intensive care unit patients (Lee et al., 2016 and Ramanathan et al., 2014) ${ }^{[10,9]}$. The patients admitted in the ICU of hospitals are most likely to receive the infections from already infected patients in the same vicinity but through different sources that may be the medical professionals who are actually trying to take care of these patients but ignored some hygienic measures.

Prevalence of UTI was found more in male samples than females. Out of 319 urinary tract infection positive samples, males samples counted 172 (approximately 54\%) while 147 (about 46\%) were from female patients. Although this difference in the prevalence of urinary tract infections between genders is not very high, it is still considerable. Both gram-negative and gram-positive bacteria cause urinary tract infections (Gajdács et al., 2020) ${ }^{[11]}$.

It was recorded in the results of the present study that urinary tract infection was found in all age groups. The youngest UTI positive patient in recorded data was just 23 years old, while the most senior UTI positive patient in ICU was 81 years of age. The prevalence percentage of urinary tract infections are shown in Fig. 3. The results of this study about the age-wise prevalence of urinary tract infections indicate that all age groups are susceptible to this problem when admitted to the intensive care unit. The results of our data revealed that maximum positive cases were found in the age group ranging from 36 years to 45 years then 46 to 55 years age group. The urinary tract infection was minimum in ICU patients with age less than 35 years. Better immunity levels and a strong body resist the maximum of upcoming diseases (Simon et al., 2015).

\section{Conclusion}

The study's objectives were to analyze the prevalence of urinary tract infections in the patients admitted in intensive care unit of different hospitals. We also aimed and observed the prevalence of urinary tract infections in different age groups and in both genders. We can summarize the results of our research in the following conclusive points.

- The prevalence of urinary tract infections is much higher in patients admitted in intensive care units than in the general population.

- Urinary tract infections may occur in both genders (males and females).

- Urinary tract infections can harm humans at any age.

\section{Recommendations}

Based on our research work and the results of the research, we can make the following recommendations to improve further research plans designed on the same grounds and to use the research for the well-being of humans practically.

- Urine samples of patients admitted in the intensive care unit should be tested for UTIs at regular intervals, after every 48 hours.

- If a urinary tract infection test is positive for a patient, further test to confirm the UTI causing uro-pathogen and its nature.

- The history of the patient should also be observed while deciding on an antibiotic for the patient.

- Age and gender must be noted to improve physician's recommendations.

- Antibiotic sensitivity tests will also help to choose the right treatment plan for a specific UTI patient.

- Hygienic conditions should be improved in intensive care units to reduce the risk factors of urinary tract infections.

\section{References}

1. Flores-Mireles AL, Walker JN, Caparon M, Hultgren SJ. Urinary tract infections: epidemiology, mechanisms of infection and treatment options. Nature reviews. Microbiology 2015;13(5):269-284. 
2. May Sewify, Shinu Nair, Samia Warsame, Mohamed Murad, Asma Alhubail, et al. Prevalence of Urinary Tract Infections and Antimicrobial Susceptibility among Diabetic Patients with Controlled and Uncontrolled Glycemia in Kuwait. Journal of Diabetes Research 2016. Article ID 6573215, 7 pages, 2016. http://doi.org/10.1155/2016/6573215

3. Alanazi MQ, Alqahtani FY, Aleanizy FS. An evaluation of $E$. coli in urinary tract infection in emergency department at KAMC in Riyadh, Saudi Arabia: retrospective study. Annals of clinical microbiology and antimicrobials 2018;17(1):3. https://doi.org/10.1186/s12941-018-0255-z

4. Jamil J, Haroon M, Sultan A, Khan MA, Gul N; Kalsoom. Prevalence, antibiotic sensitivity and phenotypic screening of ESBL/MBL producer E. coli strains isolated from urine; District Swabi, KP, Pakistan. J Pak Med Assoc 2018;68(11):1704-1707. PMID: 30410154.

5. Ullah A, Shah SRH, Almugadam BS. Prevalence of symptomatic urinary tract infections and antimicrobial susceptibility patterns of isolated uropathogens in kohat region of Pakistan. MOJ Biol Med 2018;3(3):85-89. DOI: 10.15406/mojbm.2018.03.00082

6. Liu Y, Xiao D, Shi XH. Urinary tract infection control in intensive care patients. Medicine 2018;97(38):e12195. https://doi.org/10.1097/MD.0000000000012195

7. Sotto A, De Boever CM, Fabbro-Peray P, Gouby A, Sirot D, Jourdan J. Risk factors for antibiotic-resistant Escherichia coli isolated from hospitalized patients with urinary tract infections: a prospective study. Journal of clinical microbiology 2001;39(2):438-444. https://doi.org/10.1128/JCM.39.2.438-444.2001

8. Duszyńska W, Rosenthal VD, Szczęsny A, Woźnica E, Ulfik K, Ostrowska E, et al. Urinary tract infections in intensive care unit patients - a single-centre, 3-year observational study according to the INICC project. Anaesthesiol Intensive Ther 2016;48(1):1-6. doi: 10.5603/AIT.2016.0001. PMID: 26966105.

9. Ramanathan R, Duane TM. Urinary tract infections in surgical patients. Surg Clin North Am 2014;94(6):135168. doi: 10.1016/j.suc.2014.08.007. Epub 2014 Oct 3. PMID: 25440128.

10. Eng Kiang Lee, Alvin Teo, Gillian Land, Denis Spelman, Tim Leong, Allen C Cheng. Risk factors associated with urinary tract infections in intensive care patients. Infection, Disease and Health. Published 2016, 27. DOI: https://doi.org/10.1016/j.idh.2016.03.004

11. Gajdács M, Ábrók M, Lázár A. Increasing relevance of Gram-positive cocci in urinary tract infections: a 10-year analysis of their prevalence and resistance trends. Sci Rep 2020;10:17658. https://doi.org/10.1038/s41598-02074834-y 